\title{
Médiévales
}

Langues, Textes, Histoire

76 | printemps 2019

Du nouveau en archives

\section{Rosa Maria DESSì, Les Spectres du Bon Gouvernement. Artistes, cités communales et seigneurs angevins au Trecento}

Paris, Presses universitaires de France, 2017, 444 p.

Jean-Baptiste Delzant

\section{(2) OpenEdition}

\section{Journals}

Édition électronique

URL : https://journals.openedition.org/medievales/10094

DOI : 10.4000/medievales. 10094

ISSN : $1777-5892$

\section{Éditeur}

Presses universitaires de Vincennes

Édition imprimée

Date de publication : 5 septembre 2019

Pagination : 169-171

ISBN : 978-2-37924-028-7

ISSN : 0751-2708

\section{Référence électronique}

Jean-Baptiste Delzant, «Rosa Maria dessì, Les Spectres du Bon Gouvernement. Artistes, cités

communales et seigneurs angevins au Trecento ", Médiévales [En ligne], 76 | printemps 2019, mis en ligne le 05 septembre 2019, consulté le 22 avril 2022. URL : http://journals.openedition.org/medievales/ 10094 ; DOI : https://doi.org/10.4000/medievales.10094

Ce document a été généré automatiquement le 22 avril 2022

Tous droits réservés 


\section{Rosa Maria DEsSì, Les Spectres du Bon Gouvernement. Artistes, cités communales et seigneurs angevins au Trecento}

Paris, Presses universitaires de France, 2017, 444 p.

Jean-Baptiste Delzant

\section{RÉFÉRENCE}

Rosa Maria DESSì, Les Spectres du Bon Gouvernement. Artistes, cités communales et seigneurs angevins au Trecento, Paris, Presses universitaires de France, 2017, 444 p.

1 En invoquant les spectres des peintures italiennes du XIV siècle, ces images manipulées au cours de leur longue histoire, en partie effacées et repeintes au gré des conjonctures politiques, affleurant à travers l'épaisseur des strates d'enduits, des croquis des érudits, des schémas des restaurateurs et des descriptions des écrivains, Rosa Maria Dessì présente une nouvelle lecture de l'un des cycles les plus célèbres du monde communal médiéval. Elle enrichit la compréhension d'un ensemble dont la complexité visuelle et idéologique s'offre encore à de multiples interprétations. Patrick Boucheron avait souligné, en 2013, que ces images devaient contribuer à conjurer la peur d'une seigneurie menaçant toujours d'émerger du sein même de la commune, d'une seigneurie prétendant accomplir la commune par la réduction au silence d'une conflictualité politique interne que le dominus n'aurait su juguler que par la contrainte.

2 Selon Rosa Maria Dessì, dans le palais public de Sienne, au cours du deuxième quart du $\mathrm{XIV}^{\mathrm{e}}$ siècle et en un premier état, les fresques dites du Bon Gouvernement furent conçues et réalisées par Ambrogio Lorenzetti pour exalter une paix civique établie grâce à la tutelle royale des Angevins de Naples. La peinture aurait rendu visible la façon dont la paix de la commune sortait renforcée du pacificus status institué par le 
monarque (p. 335). Le génie de l'artiste n'aurait pas d'abord résidé dans la conception d'un programme iconographique accentuant la nature idéologique d'un système politique en concentrant Commune et Bien commun en un même personnage allégorique. Maria Monica Donato, prématurément disparue (†2014) après avoir œuvré de façon considérable à la compréhension de la peinture politique des Tre et Quattrocento, avait proposé cette lecture très suggestive dès 1992. Le talent véritable de Lorenzetti fut, pour Rosa Maria Dessì, d'avoir réactivé ces images, de les avoir réajustées après la mort du roi Robert, en 1343, de sorte que la présence angevine y soit occultée et que des sens nouveaux puissent s'y déployer en faveur du régime oligarchique local. Andrea Vanni modifia à son tour le cycle après la chute du régime des Neuf, en 1355. Ce ne fut donc qu'au fil des reprises, reactationes et restaurations, très importantes au milieu du XIV ${ }^{e}$ puis au XIX ${ }^{e}$ siècle, avec un Risorgimento où les Angevins furent perçus comme une dynastie étrangère, que les images de Lorenzetti devinrent les défenseurs de l'autonomie de la commune libre de Sienne contre la tyrannie. Elles ne se figèrent que progressivement dans l'opposition aussi frontale qu'artificielle de la commune et de la seigneurie.

Développé à partir des travaux d'une habilitation à diriger les recherches, le propos est mesuré et les hypothèses avancées avec prudence. Les conclusions ne s'imposent pas moins de façon radicale. L'auteur s'inscrit dans les débats antérieurs sur les interprétations des fresques (on trouve un résumé clair des débats p. 285-293), tout en proposant deux apports majeurs. L'ouvrage dépasse alors le cas du palais siennois pour ouvrir des perspectives stimulantes jalonnées d'un corpus nourri d'œuvres peintes et sculptées du Trecento.

Les scènes peintes par Lorenzetti ne se comprennent pas hors de la domination exercée sur la Toscane par Robert d'Anjou, seigneur de Florence depuis 1313, et son fils Charles en lutte contre les partisans de l'empereur Louis de Bavière. Elles ressemblent sans les Angevins, pour paraphraser Lytton Strachey, à une représentation d'Hamlet dont le prince du Danemark serait absent. Pour autant, Rosa Maria Dessì ne propose pas une étude monographique analysant chacun des trois murs peints de la salle de la Paix dans le détail des scènes et des allégories. Le cadre de son enquête est plus large, il est celui d'une communication politique associant rituels, objets et images, mots écrits et paroles prononcées (p.99). L'auteure apporte ainsi une contribution importante au courant historiographique qui a renouvelé la perception du phénomène seigneurial dans l'Italie communale de la fin du Moyen Âge ${ }^{1}$. Républiques à dominante populaire, Florence et Sienne choisirent un temps, comme d'autres villes de la région et sans renoncer aux principes d'autonomie et de collégialité de leur propre gouvernement, de se placer sous la protection des rois français de Naples dont elles accueillirent la cour princière. Rosa Maria Dessì souligne l'étroitesse des relations qui s'établirent entre ces villes d'Italie centrale et le royaume méridional. Pour ce faire, elle suit un petit groupe d'intellectuels (juristes, prédicateurs, orateurs, poètes ou astrologues, chap. 3-4) et d'artistes (peintres et sculpteurs, chap.6) employés par le duc de Calabre ou par les cités liées à lui. Réfutant l'idée d'un antagonisme qui aurait opposé deux milieux, elle insiste sur les marques laissées dans la culture communale par la culture courtoise angevine et traque les réminiscences visuelles et thématiques de la dernière au cœur des images ornant les murs des édifices publics de Florence, Sienne ou San Gimignano (vêtements, coiffures, ornements ou signes héraldiques). 
$5 \quad \mathrm{Au} \mathrm{XV}^{\mathrm{e}}$ siècle, lorsqu'il fut demandé à Jacques d'Arras de s'en inspirer pour réaliser un ensemble de tentures, le sujet du cycle siennois fut précisé : la paix, la guerre et « le bon gouvernement du prince ». Charles de Calabre voulait incarner celui-ci, lui qui devait apporter la paix. En 1326, les factions rivales des Tolomei et des Salimbeni conclurent une concorde sous son égide: cette forme du bien commun fut ainsi acquise per lo signor, "à travers lui ", selon la formule du texte peint sous la scène du mur nord qu'interprète en ce sens R.M. Dessì en lien avec les scènes de pacification qui le surmontent. Le mauvais prince, le tyran, n'apportait lui qu'injustice et discorde : il fut figuré sur un autre mur, dans un décor que l'auteur propose de voir comme une représentation de la ville de Grosseto (p. 325-332). Longtemps dirigée par un seigneur gibelin, elle dut faire acte de soumission à Sienne en 1338. La vue de la cité dévastée serait une peinture infamante, liée à une conjoncture précise qui aurait été débordée pour devenir simultanément, dans des significations entrelacées, l'allégorie néfaste du régime tyrannique lui-même.

6 L'auteure enracine la lecture des images dans un contexte serré, minutieusement élaboré à différentes échelles. Elle a constitué pour ce faire un corpus diversifié de chroniques, recueils de sermons ou de discours, et actes de la pratique. Pour une bonne part inédites, ces sources sont issues d'un important travail en archives. Les traces qu'elles constituent sont parfois si ténues que l'argumentation semble progresser sur un fil : lorsqu'elle s'achève, le lecteur n'en porte pas moins un regard revivifié sur le paysage qu'il vient de traverser. L'une des difficultés qu'il aura rencontrées aura été de faire tenir l'ensemble des éléments proposés dans une image dont la chronologie ne peut être fixée. Des horoscopes d'un Cecco d'Ascoli brûlé en 1327 à l'ambassade d'un Giotto di Bondone intervenant lors la soumission de Grosseto, en passant par les entrées princières et les pacifications de 1326, il est délicat d'apprécier l'empreinte d'événements répartis sur une quinzaine d'années dans une seule réalisation graphique. Mais ce qui aide le lecteur, c'est précisément d'avoir renoncé à l'idée de l'unicité d'une œuvre intangible.

7 Développant des propositions qu'elle avait formulées en 2007 autour des usages du palimpseste dans la salle de la Paix, Rosa Maria Dessì demande que les images soient observées dans le flux d'états successifs qui se fondent plus qu'ils ne s'enchaînent, et non dans la stabilité idéale qui aurait suivi une exécution première voulue définitive. Si les peintures infamantes étaient peintes pour être effacées une fois purgée la peine du condamné, les ensembles monumentaux à vocation politique étaient eux aussi réalisés pour être régulièrement modifiés, partiellement effacés et repeints afin qu'ils élaborassent un nouveau message en lien avec la dernière actualité. Au fil des mises à jour, des tensions apparaissaient entre plusieurs parties de l'image, des dissonances naissaient entre des personnages et les textes peints les accompagnant qui furent eux aussi réécrits. Rosa Maria Dessì le prouve pour Sienne par l'étude des transcriptions anciennes (celle d'un manuscrit de la Marciana, du xve siècle, publiée p. 304-306) et l'examen de la syntaxe, de la métrique et de la sémantique qui éclaire la nature hétérogène des tituli formant aujourd'hui la chanson du bon gouvernement. En ce qui concerne les images, la pratique est mise en lumière grâce à l'analyse d'une série de saints angevins rapidement remaniés après leurs réalisations dans les églises et les palais publics d'Italie centrale. Le plus saisissant de ces spectres du Trecento, ces peintures manipulées, est sans doute celui de la Vierge réalisée au milieu des années 1330 par Lorenzetti à Montesiepi. Une restauration contemporaine a choisi de rendre 
visible un bras, devenu le troisième de Marie portant l'Enfant alors qu'un repeint médiéval l'avait dissimulé pour changer l'identité du personnage initial, en trône et muni des signes de la souveraineté. À Sienne, l'homme barbu et chenu en majesté aurait pu avoir été, en premier lieu, un Angevin, peut-être Robert ou son fils. Selon un processus de «transfert d'autorité figuratif » (p. 236) comparable à celui de Montesiepi, un ensemble d'accessoires et de repeints aurait permis de le transformer en une représentation de la Commune souveraine après le naufrage des ambitions de la dynastie française. Le geste s'inscrivait pleinement dans une région qui, à Sienne et Florence tout d'abord, mais aussi à Arezzo, au deuxième quart du Trecento, vit se développer un lexique politique visuel complexe, tout à la fois signe et instrument des réflexions et des pratiques du gouvernement urbain autonome.

8 L'approche de l'ouvrage peut déstabiliser. Rosa Maria Dessì prend le parti du détour pour suivre ses protagonistes les uns après les autres, de Naples à la Toscane. Elle place le lecteur dans le mouvement d'images transformées à la chronologie incertaine. À la fin du développement, elle a fortement contribué à la compréhension d'une Italie communale marquée par la présence angevine. Elle a aidé à renouveler la compréhension des célèbres fresques siennoises et, au-delà de celles-ci, à lire les images sculptées ou peintes du passé en laissant place aux temporalités multiples qui les ont façonnées.

\section{NOTES}

1. Notamment: A. zoRzI dir., Le signorie cittadine in Toscana. Esperienze di potere e forme di governo personale (sec. XIII-XV), Rome, 2013.

\section{AUTEURS}

JEAN-BAPTISTE DELZANT

Université d'Aix-Marseille 